\title{
Intraoperative Hypotension and Myocardial Infarction Development Among High-Risk Patients Undergoing Noncardiac Surgery: A Nested Case-Control Study
}

\author{
Hallqvist L, Granath F, Fored M \& Bell M. Anesthesia Analgesia 2021; 133:6-15
}

$\mathrm{D}$ ie hämodynamischen Auswirkungen im Rahmen von Allgemein- und rückenmarksnahen Regionalanästhesien sind hinreichend bekannt. Hinzu kommen Blutverluste, Flüssigkeitsverschiebungen und inflammatorische Prozesse, die den Organismus belasten. Die negativen Auswirkungen intraoperativer Hypotensionen auf den perioperativen Outcome wurden in zahlreichen Untersuchungen dargestellt.

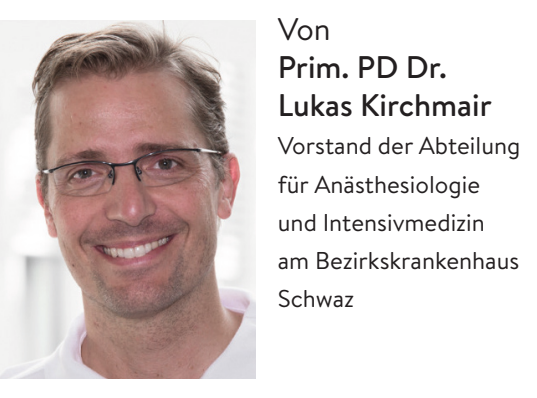
Vor allem Risikopatient*innen sind an dieser Stelle besonders gefährdet. Hauptbetroffen von einer intraoperativen Minderperfusion sind das kardiovaskuläre System, die Nieren sowie das ZNS. Es existieren jedoch unterschiedliche Definitionen einer intraoperativen Hypotension. Individualisierte Blutdruckgrenzen scheinen besser mit dem Risiko einer Organdysfunktion zu korrelieren.

Hallqvist et al. untersuchten im Rahmen der vorliegenden Studie den Zusammenhang zwischen intraoperativen Hypotensionen und myokardialen Ischämien an Hochrisikopatient*innen nach nicht kardiochirurgischen Eingriffen. Myokardiale Ischämien bzw. Myokardinfarkte (MI) gehen mit einer erhöhten perioperativen Mortalität einher. Pathogenetisch lassen sich in diesem Zusammenhang zwei Mechanismen ableiten: Typ $1 \mathrm{Ml}$ entstehen durch Plaqueruptur bzw. -erosion; Typ $2 \mathrm{Ml}$ sind bedingt durch ein Missverhältnis von Sauerstoffangebot und - bedarf. Eine Koronardissektion wird ebenfalls dem Typ $2 \mathrm{Ml}$ zugerechnet. Zu den Auslösern eines Typ $2 \mathrm{Ml}$ zählen sämtliche Mechanismen, die in der perioperativen Phase auftreten können (Arrhythmien, resp. Insuffizienz/Hypoxie, Anämie, Hypotonie, Schock).

Die Studie ging der Frage nach, ob eine intraoperative Hypotension $(\mathrm{IOH})$ einen unabhängigen Risikofaktor für

Tabelle: Risikoeinstufung für das Auftreten eines perioperativen $\mathrm{MI}$

\begin{tabular}{|c|c|}
\hline Risiko & Kriterien \\
\hline 1) sehr gering & $\begin{array}{l}\text { < } 65 \text { Jahre, ASA I, kleinere Eingriffe, } \\
\text { keine kardiovaskulären Erkrankungen }\end{array}$ \\
\hline 2) gering & $\begin{array}{l}\text { wie unter } 1 \text { ), jedoch mit } 2 \text { oder } \\
3 \text { Faktoren von 3) }\end{array}$ \\
\hline 3) moderat & $\begin{array}{l}\text { 65-79 Jahre, ASA II, mittlere Ein- } \\
\text { griffe, kardiovaskuläre Erkrankungen } \\
\text { ohne } \mathrm{MI} \text { in der Anamnese, DM }\end{array}$ \\
\hline 4) hoch & $\begin{array}{l}\text { wie unter } 3 \text { ), jedoch mit } 2 \text { oder } \\
3 \text { Faktoren von } 5 \text { ) }\end{array}$ \\
\hline 5) sehr hoch & $\begin{array}{l}\text { > } 80 \text { Jahre, ASA > II, Hochrisiko- } \\
\text { eingriffe, kardiovaskuläre Erkran- } \\
\text { kungen mit } \mathrm{MI} \text { in der Anamnese }\end{array}$ \\
\hline
\end{tabular}

das Auftreten eines perioperativen Ml darstellt. Eingeschlossen wurden Patient*innen nach nicht kardiochirurgischen Eingriffen (Ausnahme: Geburtshilfe, ambulante Eingriffe), die innerhalb von 30 Tagen postoperativ einen MI entwickelten. Die Autor*innen erhoben die Häufigkeiten von Typ-1- und Typ-2-Infarkten sowie von zugrunde liegenden intraoperativen Ereignissen (Tachykardie, Hypoxie, Blutverlust, Hb-Abfall). Eine $\mathrm{IOH}$ wurde definiert als mindestens ein systolischer Blutdruckabfall > 5 Minuten $(\leq 20 \mathrm{mmHg}, 21-40 \mathrm{mmHg}$, $41-50 \mathrm{mmHg},>50 \mathrm{mmHg}$ ). Die Studie wurde im Sinne einer Case-Control Study an drei schwedischen Kliniken durchgeführt. Insgesamt wurden 326 Patient*innen eingeschlossen und mit entsprechenden Kontrollen „gematcht“. Das perioperative Risiko der inkludierten Fälle und ihrer Kontrollen wurde wie folgt stratifiziert (siehe Tabelle):

Zusammengefasst lässt sich aus der Arbeit von Hallqvist et al. ableiten, dass ein Abfall des systolischen Blutdrucks im Ausmaß von > 50 mmHg über einen Zeitraum länger als 5 Minuten eine Vervielfachung des Risikos für einen postoperativen MI bei Hochrisikopatient*innen (OR 22) bedeutet. Aber auch geringer ausgeprägte Blutdruckabfälle gehen mit einer beachtlichen Risikoerhöhung einher. Das Bemerkenswerte an dieser Arbeit ist der immense Datenpool, auf den die Autor*innen zurückgreifen konnten. Als einer der limitierenden Faktoren ist der Umstand anzuführen, dass kardiale Biomarker nicht routinemäßig erhoben wurden und somit ausschließlich diagnostizierte MI (gemäß Definition von ESC/AHA) erfasst wurden. Stumme Ischämien, die lediglich durch eine Enzymauslenkung auffallen, wurden somit nicht detektiert und nicht in die Analyse aufgenommen.

FAZIT für die Praxis: Eine einheitliche Definition für eine $\mathrm{IOH}$ existiert immer noch nicht. Auch wenn in den letzten Jahren zahlreiche Arbeiten zur intraoperativen Blutdrucksteuerung publiziert wurden, die den systolischen Blutdruck als Zielgröße festlegten, wird in der Praxis nach wie vor der mittlere arterielle Blutdruck als relevanteste Größe angesehen. So kann ein systolischer Blutdruckabfall um $50 \mathrm{mmHg}$ immer noch in einem mittleren arteriellen Druck $>70 \mathrm{mmHg}$ resultieren. Diese und weitere Arbeiten zum Thema legen uns schon seit längerem die Betrachtung des individuellen (!) systolischen Blutdrucks im Rahmen einer intraoperativen Kreislauftherapie nahe. Als Ausgangswert soll ein unter normalen Bedingungen gemessener Wert herangezogen werden (zum Beispiel Normalstation). Wie eingangs erwähnt, sind neben dem kardiovaskulären System weitere Organsysteme von einer intraoperativen Minderperfusion betroffen. 